\title{
Proton Decay in Minimal Supersymmetric SU(5)
}

\author{
Borut Bajc ${ }^{(1)}$, Pavel Fileviez Perez ${ }^{(2)}$, and Goran Senjanović( ${ }^{(3)}$ \\ (1) J. Stefan Institute, 1001 Ljubljana, Slovenia \\ ${ }^{(2)}$ Max-Planck Institut für Physik (Werner Heisenberg Institut), Föhringer Ring 6, 80805 \\ München, Germany \\ (3) International Centre for Theoretical Physics, Trieste, Italy
}

\begin{abstract}
We systematically study proton decay in the minimal supersymmetric $\mathrm{SU}(5)$ grand unified theory. We find that although the available parameter space of soft masses and mixings is quite constrained, the theory is still in accord with experiment.
\end{abstract}

\section{INTRODUCTION}

It has been known for more than ten years that the low energy supersymmetry is tailor fit for grand unification: with the desert assumption the gauge couplings of the supersymmetric standard model unify at the single scale $M_{G U T} \approx 10^{16} \mathrm{GeV}$. Actually, this was foreseen some twenty years ago [1,2, 3, 4. However, it was noticed almost immediately that supersymmetric GUTs [5,6] carry a potential catastrophe of new $d=5$ contributions to the proton decay. This has been studied on and off for the last twenty years (see for example [7], [8]) with the culminating conclusion [9] that the minimal supersymmetric $\mathrm{SU}(5)$ theory is actually ruled out precisely due to the $d=5$ proton decay. To us ruling out the minimal theory is almost a death blow to the idea of grand unification. It is hard enough to verify the predictions of the minimal GUT; the extended versions of the theory unfortunately stop being predictive. For example, the beauty of matter unification and the naturalness of the see-saw mechanism [10 make a minimal SUSY SO(10) theory [11 more appealing. However, this is a typical example of what we are saying: the theory connects different mass scales, but does not predict them.

In view of the above it is of extreme importance to be completely sure that the minimal SUSY GUT is ruled out. This has prompted us to re-investigate this issue in gory detail. According to us, any rumor of the death of the theory is somewhat premature. More precisely, we study proton decay with arbitrary soft masses and fermion and sfermion mixings and find out the following: the model parameter space is quite constrained but not yet in contradiction with experiment. In other words, the improved measurements of proton decay will provide information about the nature of supersymmetry breaking (i.e. the soft masses) and the fermionic mass textures. This is the sector of the theory completely orthogonal to grand unification and therefore we advocate the point of view that proton decay is not yet a good test of the generic properties of grand unification (here we mean obviously the 
dimension 5 aspect of it). We should stress here that the so called decoupling regime seems to be both necessary and sufficient to save the theory from being ruled out.

In short, although we follow 9] in accepting the decoupling of the first two generations of sfermions, we cannot agree on this not being enough. The point is that we know nothing about individual fermion and sfermion mixings. Thus, proton decay simply limits these parameters and, admittedly, the restrictions are quite severe. In all honesty, it is hard to imagine a simple scenario of SUSY breaking which could be in accord with our constraints. However, a phenomenological study must always be separated from theoretical bias and, phenomenologically speaking, the theory is still alive.

\section{THE MINIMAL SUPERSYMMETRIC SU(5)}

Before starting any discussion of proton decay, one must enter the subtle issue of defining a minimal SU(5) theory. Obviously, a reasonable definition should be based on choosing a minimal Higgs sector which contains an adjoint 24 and a pair of $\mathbf{5}$ and $\overline{\mathbf{5}}$ representations.

We will show at the end of the day that even this theory (as incomplete as it is) is not in conflict with the proton decay experiment. In order to be as general as possible we perform our calculations for arbitrary values of the parameters of the theory.

In minimal $\mathrm{SU}(5)$ we can most generally write (in the renormalizable limit) for the relevant terms in the superpotential of the Higgs and Yukawa sectors

$$
\begin{gathered}
W_{H}=\frac{m_{\Sigma}}{2} \operatorname{Tr} \Sigma^{2}+\frac{\lambda}{3} \operatorname{Tr} \Sigma^{3}+\eta \overline{5}_{H} \Sigma 5_{H}+m_{H} \overline{5}_{H} 5_{H}, \\
W_{Y}=5_{H} 10^{T} Y^{U} 10+\overline{5}_{H} 10^{T} Y^{D} \overline{5}
\end{gathered}
$$

where $\Sigma$ is the $\mathrm{SU}(5)$ adjoint, $5_{H}$ and $\overline{5}_{H}$ are the Higgs fundamental and anti-fundamental superfield representations, the 10 and $\overline{5}$ refer to the three generations of matter superfields, and $Y^{\prime} s$ are $3 \times 3$ Yukawa matrices.

In the supersymmetric standard model language the Yukawa sector can be rewritten as

$$
\begin{aligned}
W_{Y}= & H Q^{T} Y_{U} u^{c}+\bar{H} Q^{T} Y_{D} d^{c}+\bar{H} e^{c T} Y_{E} L \\
+ & T Q^{T} \underline{A} Q+T u^{c T} \underline{B} e^{c}+\bar{T} Q^{T} \underline{C} L+\bar{T} u^{c T} \underline{D} d^{c},
\end{aligned}
$$

where except for the heavy triplets $T$ and $\bar{T}$ the rest are the MSSM superfields in the usual notation. The generation matrices $Y_{U, D, E}$ and $\underline{A}, \underline{B}, \underline{C}$ and $\underline{D}$ can in principle be arbitrary. In the minimal $\mathrm{SU}(5)$ defined above one finds the usual relations $\underline{A}=\underline{B}=Y_{U}=Y_{U}^{T}$, and $\underline{C}=\underline{D}=Y_{D}=Y_{E}$ at the GUT scale. The above definition of minimality implies no new structure at all energies up to $M_{P l}$. On the other hand, the lepton-down quark relations can be easily corrected by higher dimensional operators without introducing any new field at $M_{G U T}$. We postpone the discussion of higher dimensional operators for the summary and outlook.

As we mentioned before, we do not assume any specific values for the soft mass matrices of squarks and sleptons. However, as emphasized clearly in [9], we can not have all three generations of squarks contribute to the proton decay. The simplest direction to take as 
99 already did, is to assume the so called decoupling limit for the sfermions: the first two generations have a mass of order $10 \mathrm{TeV}$, thus effectively decoupling from the rest, while the third is of order $1 \mathrm{TeV}$ [12,13, 14]. This is still in accord with naturalness constraints and the limits from flavour violation in neutral current phenomena suggest small mixings with the first two generations of fermions. We will see later that it is possible to make the proton decay be in agreement with experiment, again for some combinations of such mixings being small.

With this in mind we allow the mass diagonalization matrices to be different for particles and sparticles. For the fermions we have

$$
\begin{aligned}
U^{T} Y_{U} U_{c} & =Y_{U}^{d}, \\
D^{T} Y_{D} D_{c} & =Y_{D}^{d}, \\
E_{c}^{T} Y_{E} E & =Y_{E}^{d},
\end{aligned}
$$

where $X\left(X_{c}\right)$ is the unitary matrix that rotates the fermion $x\left(x^{c}\right)$ from the flavour to the mass basis. The only combination we know from low-energy experiments is $U^{\dagger} D=V_{C K M}$ (and a similar one in the lepton sector, $N^{\dagger} E=V_{l}$, the leptonic mixing matrix).

Similarly, the unitary matrices $\tilde{X}\left(\tilde{X}_{c}\right)$ rotate the bosons $\tilde{x}\left(\tilde{x}^{c}\right)$ from the flavour to the mass states. Once $\mathrm{SU}(2)_{L}$ is spontaneously broken, there is also in general a nonzero mixing between the bosonic states $\tilde{x}$ and $\left(\tilde{x}^{c}\right)^{*}$ : their relative importance is proportional to $m_{W} / m_{\tilde{f}}$, which is, for our choice of the squark and slepton masses, not bigger than 1/10. We assume this to be small enough to consider it as a perturbation.

The calculation itself is tedious but straightforward, and thus we leave the details for the Appendix. We simply turn to the systematic analysis of the possible solutions which keep proton stable enough.

\section{WHY PROTON DECAY DOES NOT RULE OUT MINIMAL SU(5)}

In this central section of our paper (the only one you should read if you just wish to get our main point) we stick to the very minimal SUSY SU(5) theory. In other words we assume the conditions discussed above (valid when $M_{P l} \rightarrow \infty$ ) in the theory with only 5 and $\overline{5}$ light Higgses:

$$
\begin{aligned}
& \underline{A}=\underline{B}=Y_{U}=Y_{U}^{T}, \\
& \underline{C}=\underline{D}=Y_{D}=Y_{E},
\end{aligned}
$$

where, of course, these conditions are valid at the unification scale. A quick glance at the Appendix shows that the longevity of the proton can be achieved by, say, the following conditions at $1 \mathrm{GeV}$ :

$$
\begin{aligned}
\left(\tilde{U}^{\dagger} D\right)_{31,32} & \approx 0, \\
\left(\tilde{D}^{\dagger} D\right)_{31,32} & \approx 0, \\
\left(\tilde{U}_{c}^{T} Y_{U}^{T} D\right)_{31,32} & \approx 0, \\
\left(\tilde{N}^{T} \underline{C}^{T} D\right)_{31,32}\left(\tilde{U}^{T} \underline{A} D\right)_{32,31} & \approx 0, \\
\left(\tilde{E}_{c}^{\dagger} E_{c}\right)_{31,32} & \approx 0,
\end{aligned}
$$




$$
\begin{aligned}
\left(\tilde{D}_{c}^{\dagger} D_{c}\right)_{31,32} & \approx 0, \\
\left(\tilde{E}^{\dagger} E\right)_{31,32} & \approx 0, \\
\left(\tilde{N}^{\dagger} E\right)_{31,32} & \approx 0 .
\end{aligned}
$$

If one wishes to quantify these conditions, one can not take (5)-(6) at face value, but instead must compute the departure due to the running from $M_{G U T}$ to $1 \mathrm{GeV}$. It makes no sense to do this here; after all, this is just a prototype example and it can surely be satisfied at any scale.

In the above equations we simply mean that all the terms must be small. How small? It is hard to quantify this precisely and, honestly speaking, it seems to us a premature task. Our aim was to demonstrate that the theory is still consistent with data and from the above formulae it is obvious. If (when) proton decay is discovered and the decay modes measured, it may be sensible to see how small should the above terms be. Suffice it to say, that a percent suppression of the super KM results should be enough [9]. This means that on the average each vertex should be suppressed by a factor of $1 / 3$ or so with respect to the minimal supergravity predictions. It is very difficult to say more: in fact one could be tempted to estimate that for example the combinations on the lefthandsides of the above equations need to be at least $10^{-2}$ the same combinations in super KM. However this is not authomatically neither necessary nor enough. The fact is, that we have to do with a nonlinear system, since the total decay in a specified mode is proportional to the square of a sum of single diagrams, each of them is proportional to the product of four unknown mixings. Some of these mixings contribute to different diagrams, and some depend on others, so the task of constraining them numerically seem exagerate in view of our complete ignorance of all these parameters. What we can say for sure is that if each of the diagrams in the appendix is suppressed by a factor of $1 / 100$ with respect to the minimal supergravity predictions, proton decay is not too fast and minimal supersymmetric $\mathrm{SU}(5)$ is not ruled out.

Notice that all the terms can be made to vanish by a judicious choice of squark and slepton mixing matrices. In other words, at this point the proton decay limits provide information on the properties of sfermions and not on the structure of the unified theory.

Notice further that the so called super KM basis, in which the mixing angles of fermions and sfermions are equal, does not work for the proton decay, since eqs. (7), (9), (10) and (14) are not satisfied. If you believe in super KM, you would conclude that the theory is ruled out. It is obvious though, from our work, that this is not true in general.

Notice even further, that all the relations (7)-(14) do not require the extreme minimality conditions (5)-(6). More precisely, one can opt for the improvement of the fermion mass relations and still save the proton.

One could worry that the above constraints for the sfermion and fermion mixing matrices could be in contradiction with the experimental bounds on the flavour violation low energy processes. Fortunately, this is not true. Namely, the same conditions (77)-(14) suffice to render neutral current flavour violation in-offensive (of course, the decoupling is necessary for this to be true).

The analysis in the Appendix has been done with the assumption of no left-right sfermion, neutralino or chargino mixing. As we explained at the end of the previous section, this mixing can be included in a perturbative way: one can show that, up to two mass insertions, the same constraints (7)-(14) kill all the contributions to nucleon decay. This is enough to 
increase the nucleon lifetime above the experimental limit, since each mixing multiplies the diagram by at least $1 / 10$.

Up to now we have discussed only the $\mathrm{d}=5$ nucleon decay. What about a generic $\mathrm{d}=6$ contribution of gauge bosons relevant for both ordinary and SUSY GUTs? In the very minimal case, $Y_{D}=Y_{E}$ and $Y_{U}=Y_{U}^{T}$, this is completely determined by the CKM matrix [15. However, as soon one abandons this unrealistic situation, this is not true anymore and the individual up and down quark and lepton mixings enter the game and proton decay is not as determined as before [16, 17].

\section{SUMMARY AND OUTLOOK}

We hope to have convinced the reader that the supersymmetric $\mathrm{SU}(5)$ theory even in its very minimal version is still alive and still in accord with the nucleon decay limits. All that is required is simply small mixing angles among squarks (sleptons) and/or quarks (leptons), on top of the decoupling hypothesis, which sees the first two generations of sfermions pushed to the $10 \mathrm{TeV}$ region.

Does this mean that the proton decay experiments really probe the sfermion and fermion mixing matrices? More precisely, are there any other uncertainties involved in this game? At first glance the answer is no. After all we have carefully defined the minimal theory and found the predictions discussed above. However, two points can still be raised.

(i) Triplet-octet splitting (higher dimensional operators in the Higgs sector).

In order to appreciate this point let us discuss the origin of the problem in question. If one assumes that the heavy particles in the adjoint superfield $\Sigma$ (the color octet and the weak triplet) have the masses equal to $M_{G U T}$, the gauge couplings unify at $\approx M_{G U T} \approx 10^{16} \mathrm{GeV}$. In this case the masses of heavy triplets $T$ and $\bar{T}$ are smaller than $\approx 3.6 \times 10^{15} \mathrm{GeV}$ [9. A factor of around 20 increase of triplet masses according to [9] is sufficient to satisfy all the experimental constraints.

A simple possibility, which allows this, is to increase $M_{G U T}$ itself by a similar factor of 20 or so. This turns out to be easily satisfied by simply splitting the octet and triplet masses in $\Sigma$ and allowing them to be smaller than $M_{G U T}$ [18].

Imagine for example that the octets and triplets are light enough, so that their masses originate from dimension 4 Planck scale induced terms in the superpotential, i.e. assume that the renormalizable cubic term in the superpotential (1) is negligible. In that case $m_{3}=4 m_{8}$ [19], which at the one-loop level increases the proton decay mediating Higgs triplet masses by about a factor of 30 .

(ii) Improving the Yukawa sector with higher dimensional operators. In the minimal SU(5) theory and in the limit $M_{P l} \rightarrow \infty$ the proton decay mediating Higgs triplet couplings are set by $\mathrm{SU}(5)$ symmetry, since they must be equal to the ordinary doublet couplings (5)-(6). These relations can be, in the spirit of [20], changed by the nonrenormalizable $1 / M_{P l}$ suppressed operators [21,22,23]. This induces unfortunately additional uncertainty in the constraints for the sfermion and fermion mixings.

In other words, to us the nucleon decay not only can not rule out the structure of the theory, but even in the case of observation would not easily provide enough information about sfermion and fermion individual mixings. In any case we see no reason whatsoever 
why one should search for modifications of the theory at the GUT scale or below for the sake of proton decay. If you need to do model building, do not look here for an excuse.

\section{ACKNOWLEDGMENTS}

We are extremely grateful to Ilia Gogoladze for collaborating with us in the early stage of this work and for numerous discussions and encouragement. We thank Gia Dvali for discussions and comments, Lotfi Boubeckeur and Jure Zupan for useful suggestions and Ariel Garcia for discussions. G.S. is extremely grateful to Alexei Smirnov for strong (although indirect) encouragement to publish these results. The work of B.B. is supported by the Ministry of Education, Science and Sport of the Republic of Slovenia; the work of G.S. is partially supported by EEC under the TMR contracts ERBFMRX-CT960090 and HPRNCT-2000-00152. Both B.B. and P.F.P thank ICTP for hospitality during the course of this work.

\section{APPENDIX}

In this Appendix we present the complete set of diagrams responsible for $\mathrm{d}=5$ nucleon decay in the minimal supersymmetric SU(5) theory. In our notation $T$ and $\bar{T}$ stand for heavy Higgs triplets; $\tilde{T}$ and $\tilde{\bar{T}}$ denote their fermionic partners; $\tilde{w}^{ \pm}$stands for winos, $\tilde{h}_{+, 0}$ and $\tilde{\bar{h}}_{-, 0}$ are light Higgsinos and $\tilde{V}_{0}$ stand for neutral gauginos.

1) $\underline{p \rightarrow\left(K^{+}, \pi^{+}, \rho^{+}, K^{*+}\right) \bar{\nu}_{i}}, \underline{n \rightarrow\left(\pi^{0}, \rho^{0}, \eta, \omega, K^{0}, K^{* 0}\right) \bar{\nu}_{i}}, \quad(i=1,2,3)$

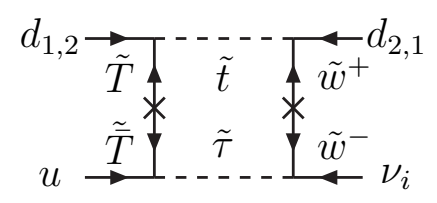

$$
\propto \quad\left(D^{T} \underline{A} \tilde{U}\right)_{13,23}\left(\tilde{U}^{\dagger} D\right)_{32,31}\left(N^{T} \tilde{E}^{*}\right)_{i 3}\left(\tilde{E}^{T} \underline{C}^{T} U\right)_{31}
$$

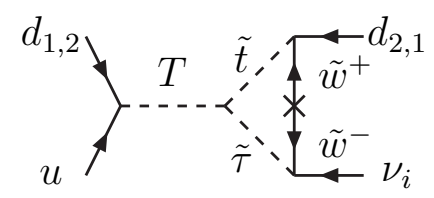

$$
\propto \quad\left(D^{T} \underline{A} U\right)_{11,21}\left(N^{T} \tilde{E}^{*}\right)_{i 3}\left(\tilde{E}^{T} \underline{C}^{T} \tilde{U}\right)_{33}\left(\tilde{U}^{\dagger} D\right)_{32,31}
$$

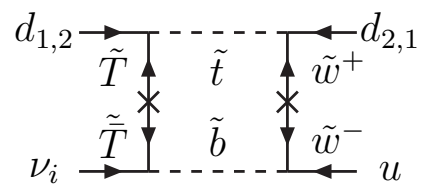

$$
\propto \quad\left(D^{T} \underline{A} \tilde{U}\right)_{13,23}\left(\tilde{U}^{\dagger} D\right)_{32,31}\left(U^{T} \tilde{D}^{*}\right)_{13}\left(\tilde{D}^{T} \underline{C} N\right)_{3 i}
$$

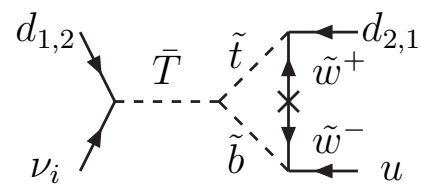

$$
\propto \quad\left(D^{T} \underline{C} N\right)_{1 i, 2 i}\left(U^{T} \tilde{D}^{*}\right)_{13}\left(\tilde{D}^{T} \underline{A} \tilde{U}\right)_{33}\left(\tilde{U}^{\dagger} D\right)_{32,31}
$$

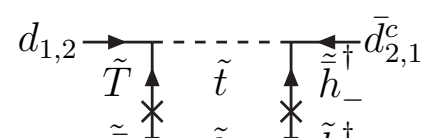

$$
\propto \quad\left(D^{T} \underline{A} \tilde{U}\right)_{13,23}\left(\tilde{U}^{\dagger} Y_{D}^{*} D_{c}^{*}\right)_{32,31}\left(U_{c}^{\dagger} Y_{U}^{\dagger} \tilde{D}^{*}\right)_{13}\left(\tilde{D}^{T} \underline{C} N\right)_{3 i}
$$




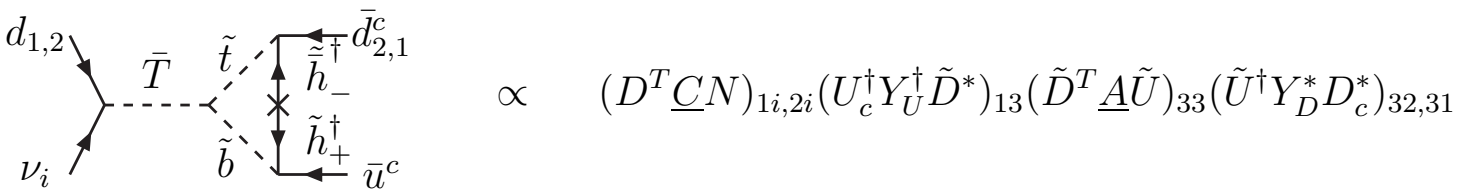

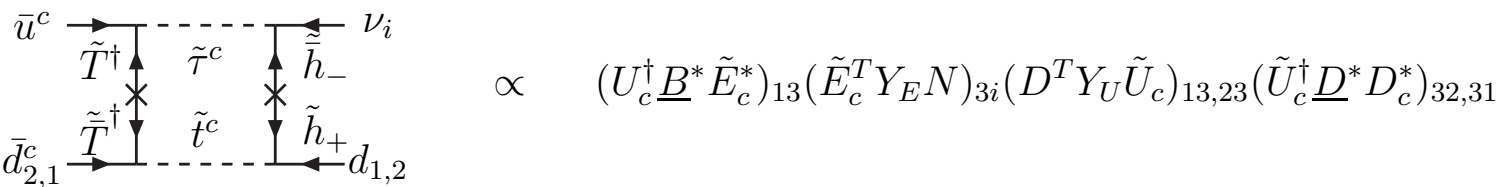

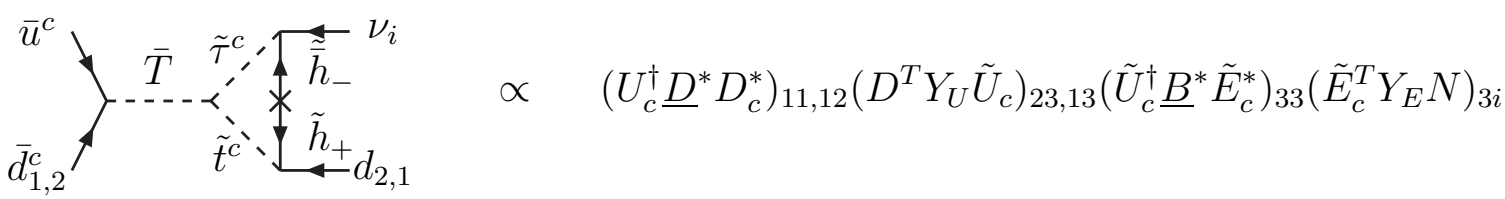

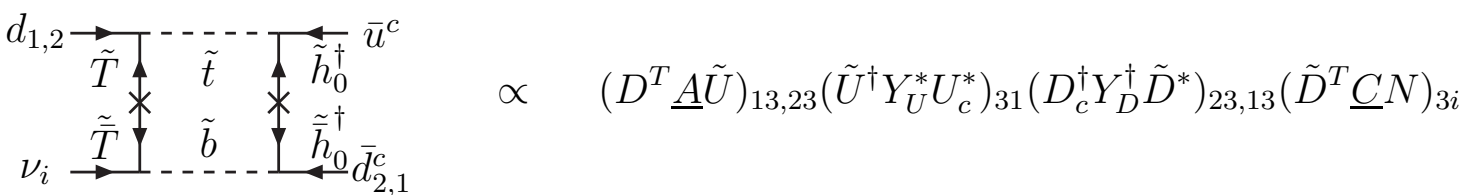

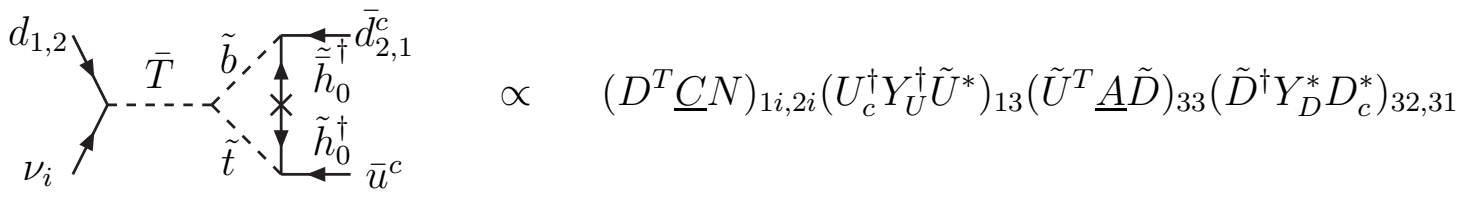

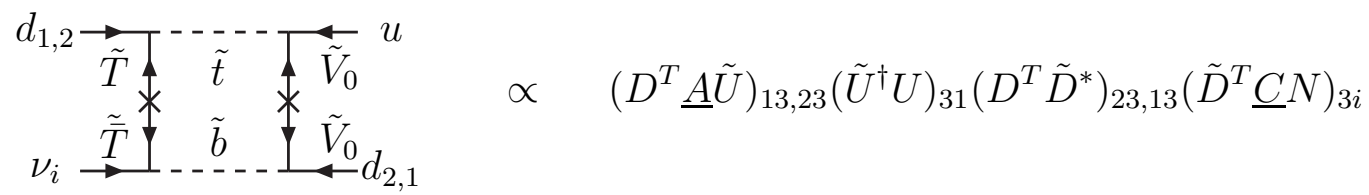

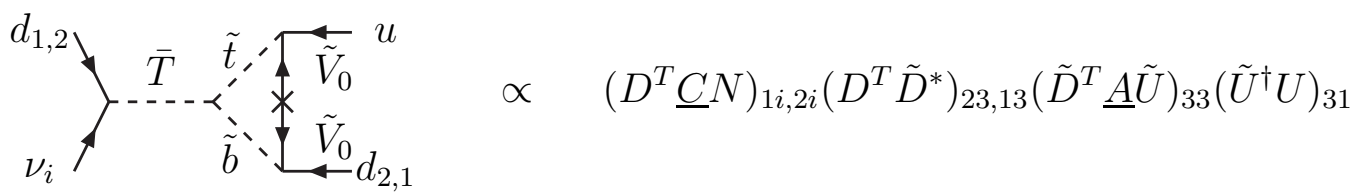

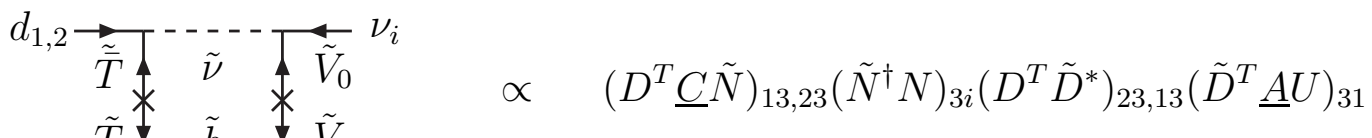

$$
\begin{aligned}
& \stackrel{\tilde{T}}{\rightarrow} \tilde{b} \underset{V_{0}}{\leftarrow} d_{2,1}
\end{aligned}
$$

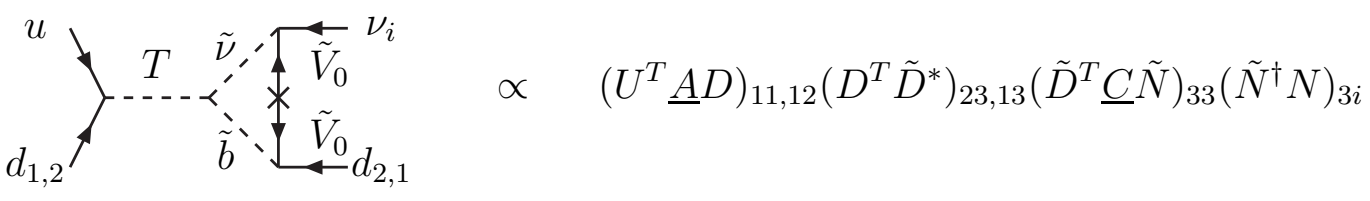

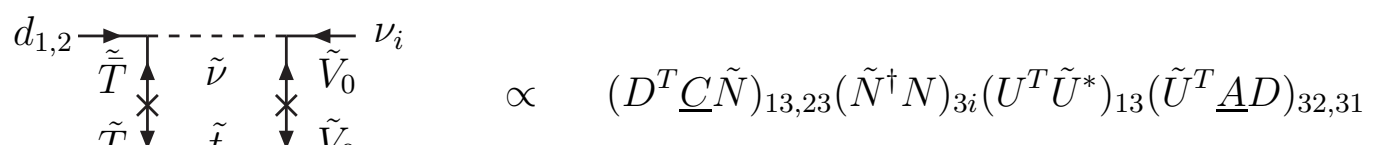


2) $\underline{p \rightarrow\left(K^{0}, \pi^{0}, \eta, K^{* 0}, \rho^{0}, \omega\right) e_{i}^{+}}, \underline{n \rightarrow\left(K^{-}, \pi^{-}, K^{*-}, \rho^{-}\right) e_{i}^{+}} \quad\left(i=1,2\right.$, for $K^{*}$ only $\left.i=1\right)$
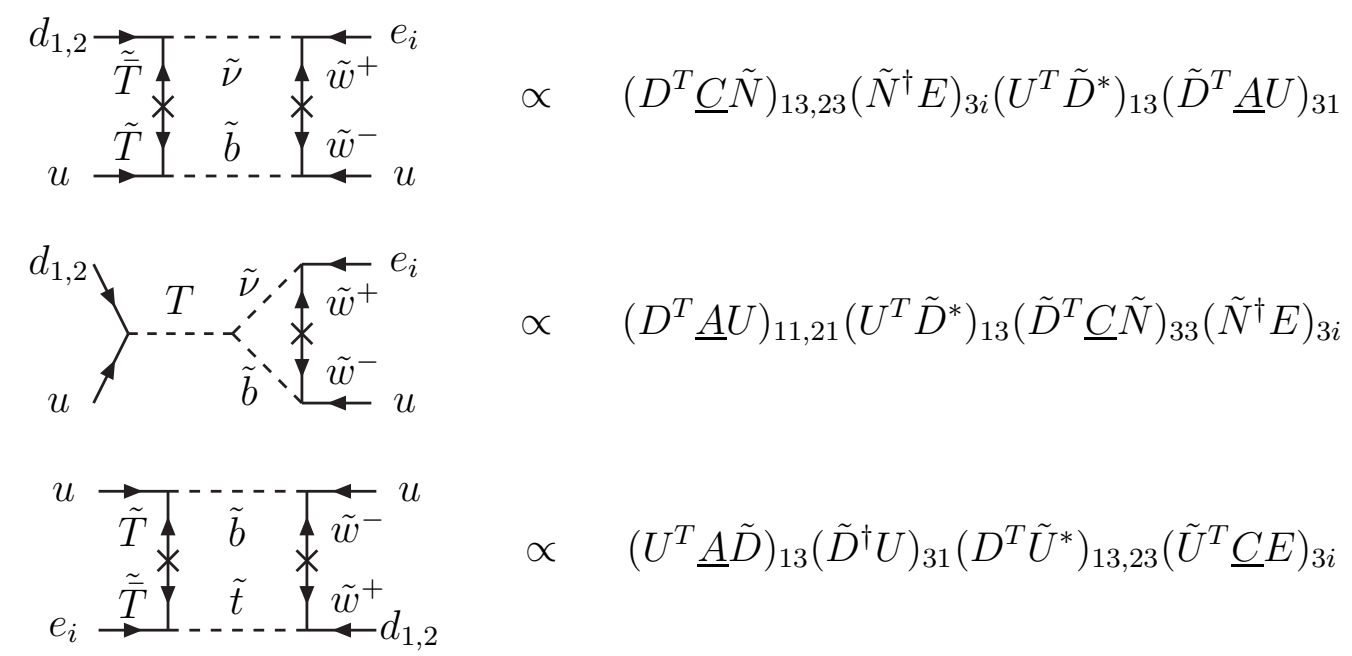

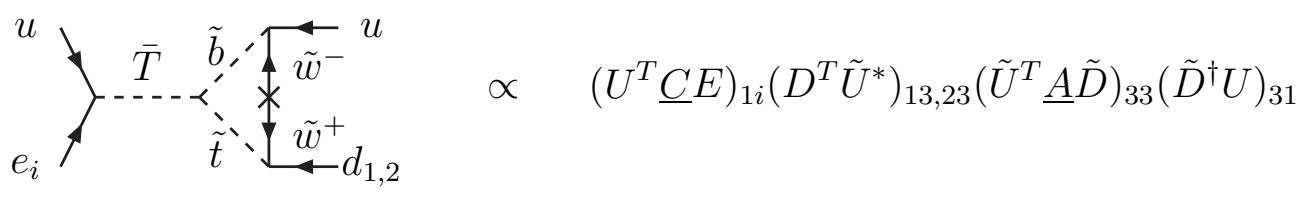

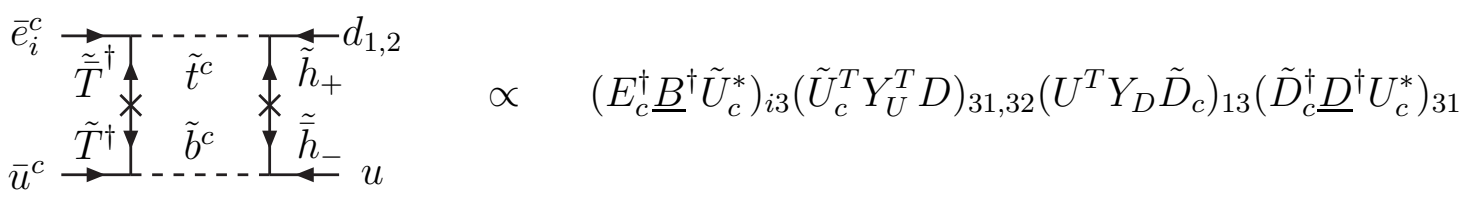

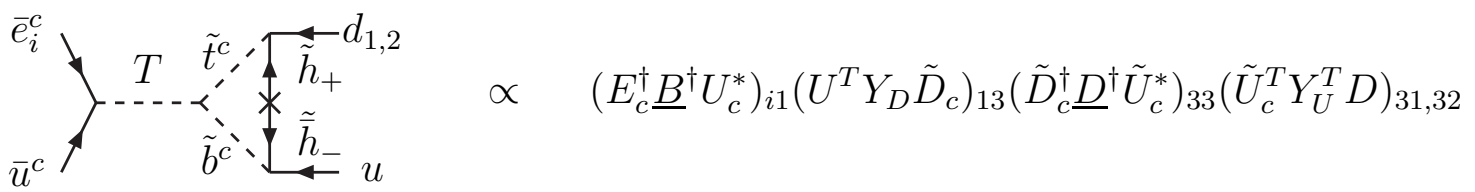

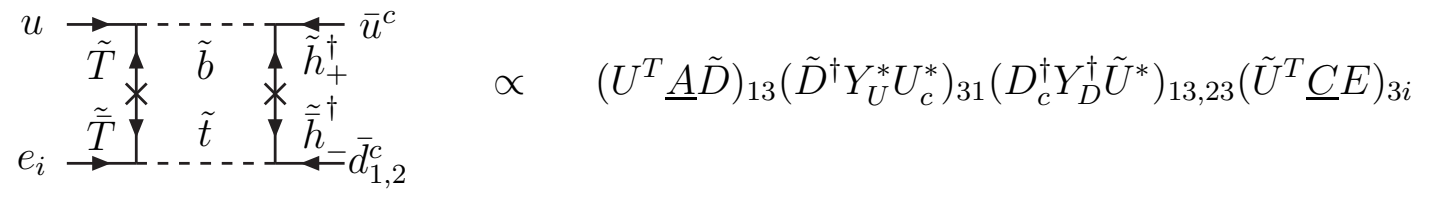

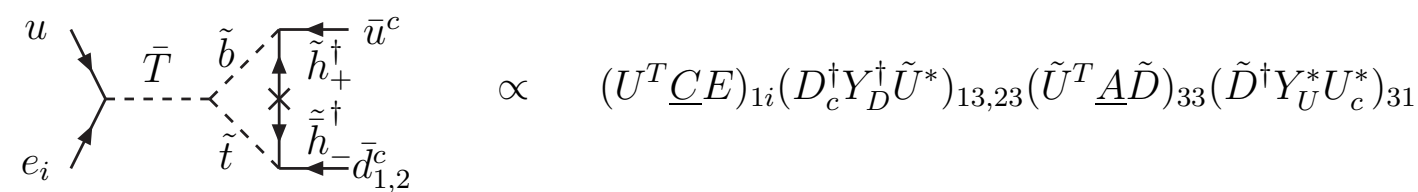

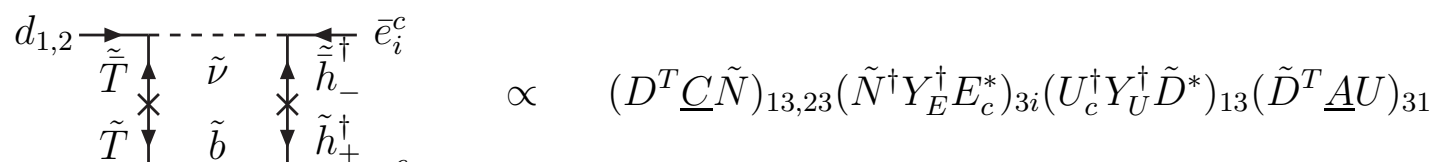

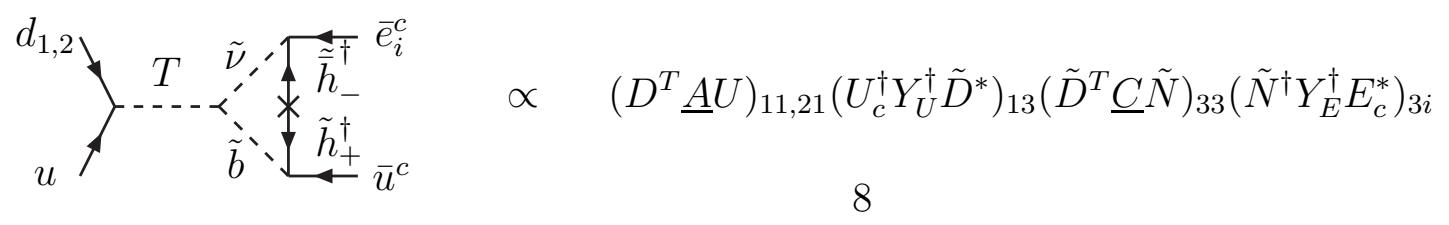




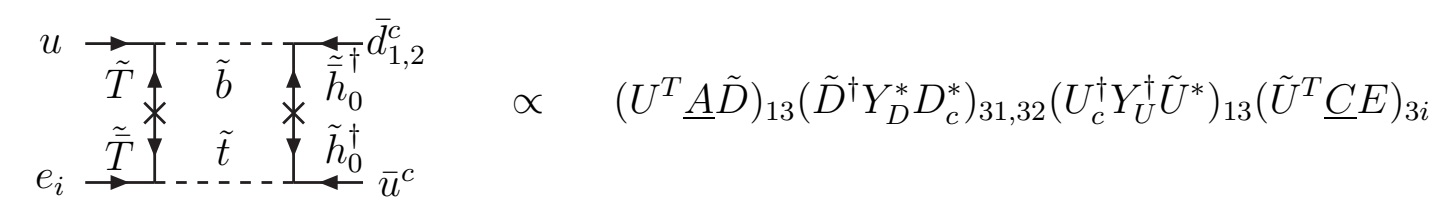

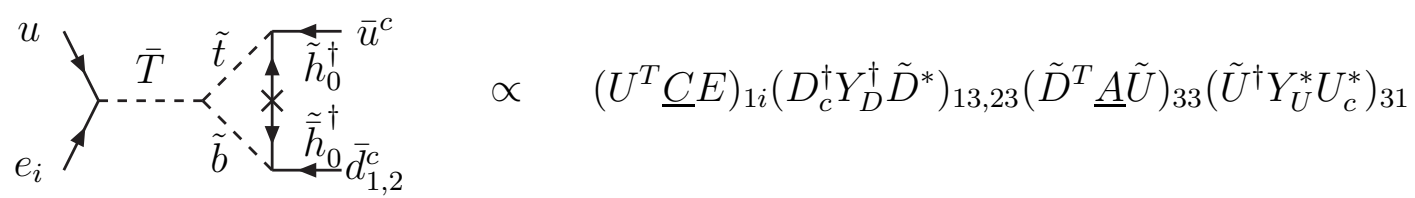

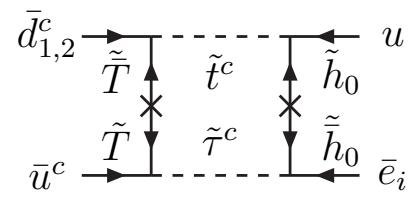

$\propto \quad\left(D_{c}^{\dagger} \underline{D}^{\dagger} \tilde{U}_{c}^{*}\right)_{13,23}\left(\tilde{U}_{c}^{T} Y_{U}^{T} U\right)_{31}\left(E^{T} Y_{E}^{T} \tilde{E}_{c}\right)_{i 3}\left(\tilde{E}_{c}^{\dagger} \underline{B}^{\dagger} U_{c}^{*}\right)_{31}$

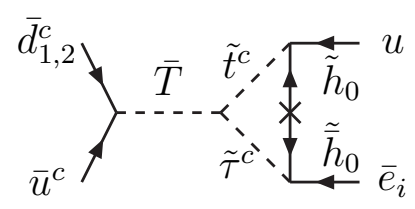

$\propto \quad\left(D_{c}^{\dagger} \underline{D}^{\dagger} U_{c}^{*}\right)_{11,21}\left(E^{T} Y_{E}^{T} \tilde{E}_{c}\right)_{i 3}\left(\tilde{E}_{c}^{\dagger} \underline{B}^{\dagger} \tilde{U}_{c}^{*}\right)_{33}\left(\tilde{U}_{c}^{T} Y_{U}^{T} U\right)_{31}$

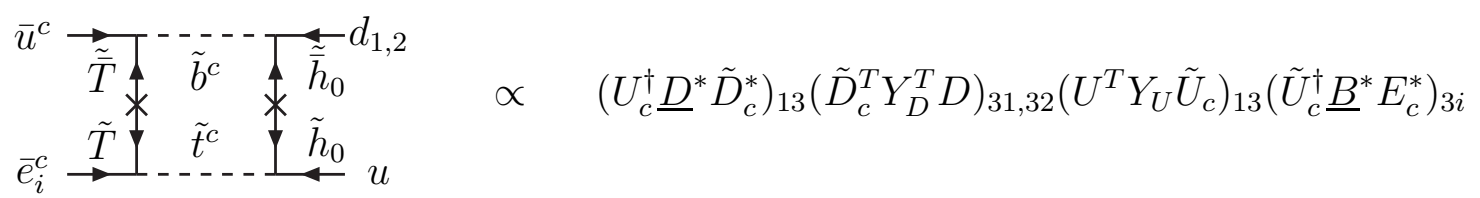

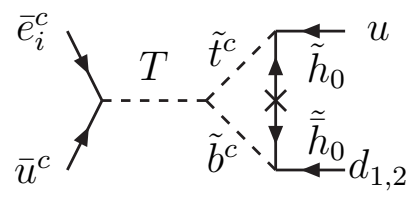

$\propto \quad\left(E_{c}^{\dagger} \underline{B}^{\dagger} U_{c}^{*}\right)_{i 1}\left(D^{T} Y_{D} \tilde{D}_{c}\right)_{13,23}\left(\tilde{D}_{c}^{\dagger} \underline{D}^{\dagger} \tilde{U}_{c}^{*}\right)_{33}\left(\tilde{U}_{c}^{T} Y_{U}^{T} U\right)_{31}$

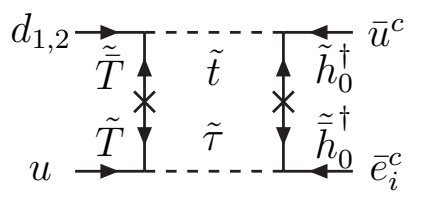

$\propto \quad\left(D^{T} \underline{A} \tilde{U}\right)_{13,23}\left(\tilde{U}^{\dagger} Y_{U}^{*} U_{c}^{*}\right)_{31}\left(E_{c}^{\dagger} Y_{E}^{*} \tilde{E}^{*}\right)_{i 3}\left(\tilde{E}^{T} \underline{C}^{T} U\right)_{31}$

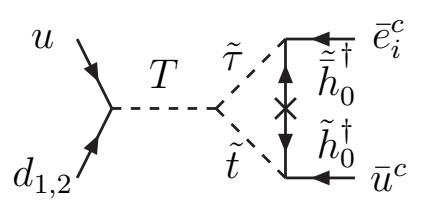

$\propto \quad\left(U^{T} \underline{A} D\right)_{11,12}\left(U_{c}^{\dagger} Y_{U}^{\dagger} \tilde{U}^{*}\right)_{13}\left(\tilde{U}^{T} \underline{C} \tilde{E}\right)_{33}\left(\tilde{E}^{\dagger} Y_{E}^{\dagger} E_{c}^{*}\right)_{3 i}$

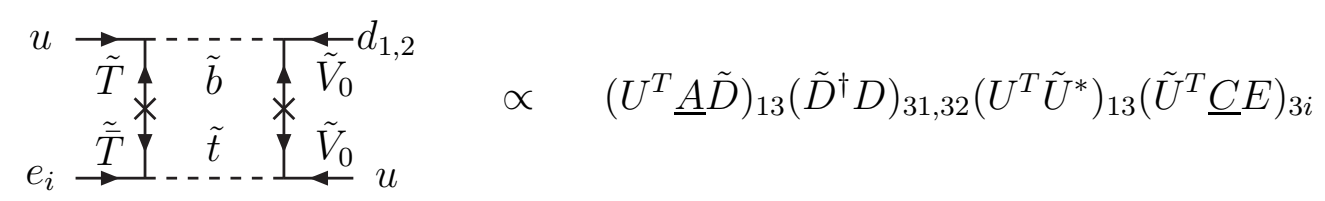

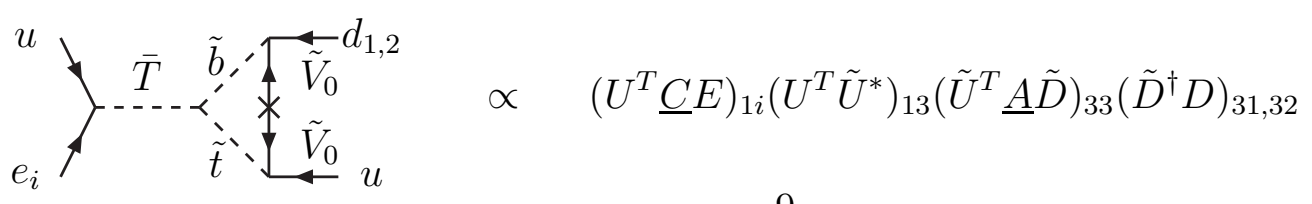



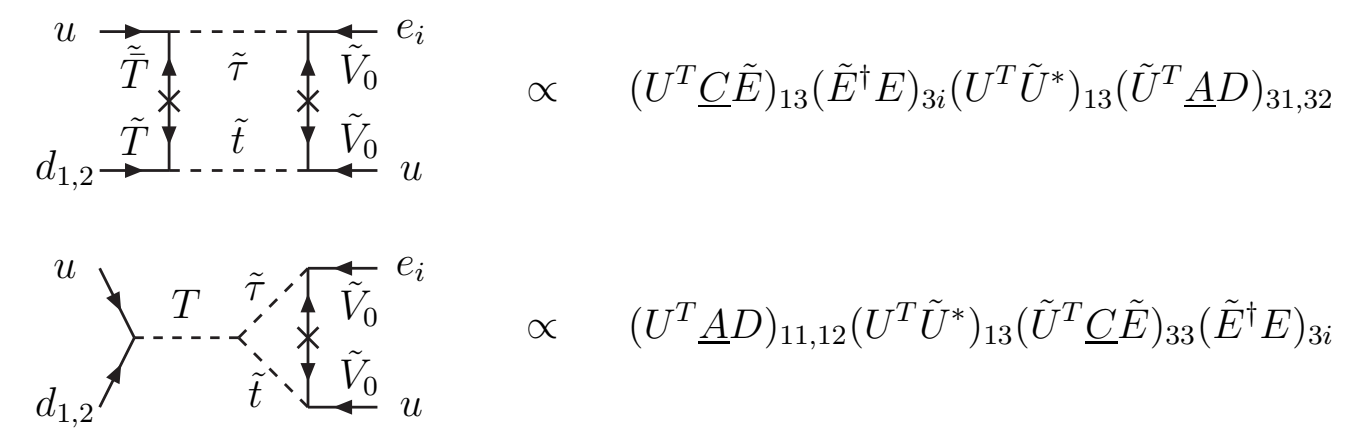

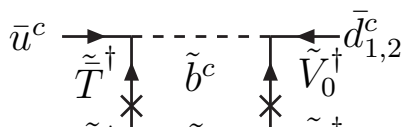
$\bar{e}_{i}^{c} \stackrel{\tilde{T}^{\dagger}}{\rightarrow} \tilde{t}^{c} \stackrel{\tilde{V}_{0}^{\dagger}}{\bar{u}^{c}}$

$\propto \quad\left(U_{c}^{\dagger} \underline{D}^{*} \tilde{D}_{c}^{*}\right)_{13}\left(\tilde{D}_{c}^{T} D_{c}^{*}\right)_{31,32}\left(U_{c}^{\dagger} \tilde{U}_{c}\right)_{13}\left(\tilde{U}_{c}^{\dagger} \underline{B}^{*} E_{c}^{*}\right)_{3 i}$

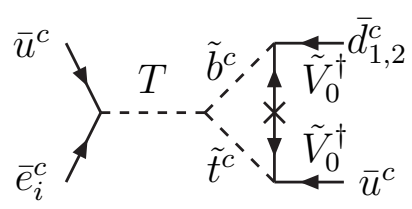

$\propto \quad\left(U_{c}^{\dagger} \underline{B}^{*} E_{c}^{*}\right)_{1 i}\left(U_{c}^{\dagger} \tilde{U}_{c}\right)_{13}\left(\tilde{U}_{c}^{\dagger} \underline{D}^{*} \tilde{D}_{c}^{*}\right)_{33}\left(\tilde{D}_{c}^{T} D_{c}^{*}\right)_{31,32}$

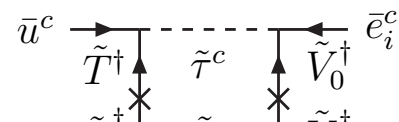
$\bar{d}_{1,2}^{c} \stackrel{\tilde{\bar{T}}^{\dagger}}{\rightarrow} \tilde{t}^{c} \underline{V}_{0}^{\dagger} \bar{u}^{c}$

$\propto \quad\left(U_{c}^{\dagger} \underline{B^{*}} \tilde{E}_{c}^{*}\right)_{13}\left(\tilde{E}_{c}^{T} E_{c}^{*}\right)_{3 i}\left(U_{c}^{\dagger} \tilde{U}_{c}\right)_{13}\left(\tilde{U}_{c}^{\dagger} \underline{D^{*}} D_{c}^{*}\right)_{31,32}$

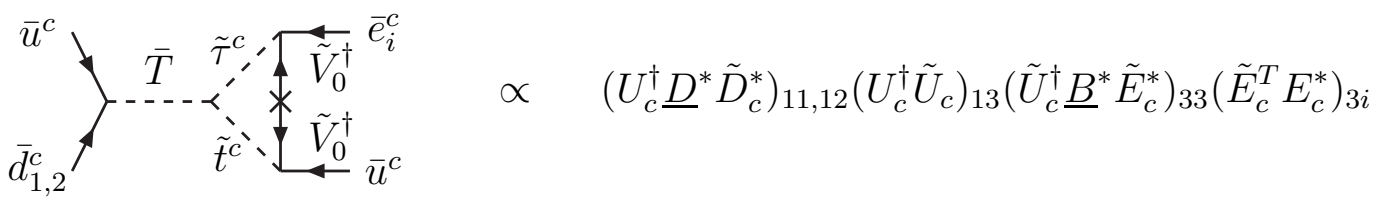




\section{REFERENCES}

[1] S. Dimopoulos, S. Raby and F. Wilczek, "Supersymmetry And The Scale Of Unification," Phys. Rev. D 24 (1981) 1681.

[2] L. E. Ibañez and G. G. Ross, "Low-Energy Predictions In Supersymmetric Grand Unified Theories," Phys. Lett. B 105 (1981) 439.

[3] M. B. Einhorn and D. R. Jones, "The Weak Mixing Angle And Unification Mass In Supersymmetric SU(5)," Nucl. Phys. B 196 (1982) 475.

[4] W. J. Marciano and G. Senjanović, "Predictions Of Supersymmetric Grand Unified Theories," Phys. Rev. D 25 (1982) 3092.

[5] N. Sakai and T. Yanagida, "Proton Decay In A Class Of Supersymmetric Grand Unified Models," Nucl. Phys. B 197 (1982) 533.

[6] S. Weinberg, "Supersymmetry At Ordinary Energies. 1. Masses And Conservation Laws," Phys. Rev. D 26 (1982) 287.

[7] J. Hisano, H. Murayama and T. Yanagida, "Nucleon decay in the minimal supersymmetric SU(5) grand unification," Nucl. Phys. B 402 (1993) 46 arXiv:hep-ph/9207279.

[8] T. Goto and T. Nihei, "Effect of RRRR dimension 5 operator on the proton decay in the minimal SU(5) SUGRA GUT model," Phys. Rev. D 59 (1999) 115009 arXiv:hep$\mathrm{ph} / 9808255]$.

[9] H. Murayama and A. Pierce, "Not even decoupling can save minimal supersymmetric SU(5)," Phys. Rev. D 65 (2002) 055009 arXiv:hep-ph/0108104.

[10] M. Gell-Mann, P. Ramond and R. Slansky, proceedings of the Supergravity Stony Brook Workshop, New York, 1979, eds. P. Van Niewenhuizen and D. Freeman (North-Holland, Amsterdam); T. Yanagida, proceedings of the Workshop on Unified Theories and Baryon Number in the Universe, Tsukuba, Japan 1979 (edited by A. Sawada and A. Sugamoto, KEK Report No. 79-18, Tsukuba); R. N. Mohapatra and G. Senjanović, "Neutrino Masses And Mixings In Gauge Models With Spontaneous Parity Violation," Phys. Rev. D 23 (1981) 165.

[11] For a recent comprehensive study of the predictions of this theory see C. S. Aulakh, B. Bajc, A. Melfo, A. Rašin and G. Senjanović, "SO(10) theory of R-parity and neutrino mass," Nucl. Phys. B 597 (2001) 89 arXiv:hep-ph/0004031.

[12] A. Pomarol and D. Tommasini, "Horizontal symmetries for the supersymmetric flavor problem," Nucl. Phys. B 466 (1996) 3 arXiv:hep-ph/9507462.

[13] G. R. Dvali and A. Pomarol, "Anomalous U(1) as a mediator of supersymmetry breaking," Phys. Rev. Lett. 77 (1996) 3728 [arXiv:hep-ph/9607383].

[14] A. G. Cohen, D. B. Kaplan and A. E. Nelson, "The more minimal supersymmetric standard model," Phys. Lett. B 388 (1996) 588 arXiv:hep-ph/9607394.

[15] R. N. Mohapatra, "Flavor Mixing In SU(5) Grand Unified Theories," Phys. Rev. Lett. 43 (1979) 893.

[16] S. Nandi, A. Stern and E. C. Sudarshan, "Can Proton Decay Be Rotated Away?," Phys. Lett. B 113 (1982) 165.

[17] V. S. Berezinsky and A. Y. Smirnov, "How To Save Minimal SU(5)," Phys. Lett. B 140 (1984) 49.

[18] C. Bachas, C. Fabre and T. Yanagida, "Natural gauge-coupling unification at the string scale," Phys. Lett. B 370 (1996) 49 arXiv:hep-th/9510094. 
[19] J. L. Chkareuli and I. G. Gogoladze, "Unification picture in minimal supersymmetric SU(5) model with string remnants," Phys. Rev. D 58 (1998) 055011 arXiv:hepph/9803335.

[20] G. R. Dvali, "Can 'doublet - triplet splitting' problem be solved without doublet - triplet splitting?," Phys. Lett. B 287 (1992) 101.

[21] P. Nath, "Hierarchies and Textures in Supergravity Unification," Phys. Rev. Lett. 76 (1996) 2218 arXiv:hep-ph/9512415.

[22] P. Nath, "Textured Minimal And Extended Supergravity Unification And Implications For Proton Stability," Phys. Lett. B 381 (1996) 147 arXiv:hep-ph/9602337.

[23] Z. Berezhiani, Z. Tavartkiladze and M. Vysotsky, " $\mathrm{d}=5$ operators in SUSY GUT: Fermion masses versus proton decay," arXiv:hep-ph/9809301. 